\title{
Editorial: Magnetohydrodynamic Waves in the Solar Atmosphere: Heating and Seismology
}

\author{
Tom Van Doorsselaere ${ }^{1 *}$, Valery M. Nakariakov ${ }^{2,3}, \mathrm{Bo} \mathrm{Li}^{4}$ and Patrick Antolin ${ }^{5,6}$ \\ ${ }^{1}$ Department of Mathematics, Centre for mathematical Plasma Astrophysics, KU Leuven, Leuven, Belgium, ${ }^{2}$ Department of \\ Physics, Centre for Fusion, Space and Astrophysics, University of Warwick, Coventry, United Kingdom, ${ }^{3}$ St. Petersburg \\ Branch, Special Astrophysical Observatory, Russian Academy of Sciences, St. Petersburg, Russia, ${ }^{4}$ School of Space \\ Science and Physics, Shandong University, Weihai, China, ${ }^{5}$ Department of Mathematics, Physics and Electrical Engineering, \\ Northumbria University, Newcastle upon Tyne, United Kingdom, ${ }^{6}$ School of Mathematics and Statistics, University of \\ St. Andrews, St. Andrews, United Kingdom
}

Keywords: solar physics, MHD waves, solar corona, solar coronal seismology, coronal heating

Editorial on the Research Topic

Magnetohydrodynamic Waves in the Solar Atmosphere: Heating and Seismology

Historically, following the seminal paper on what is now called "Alfvén" waves (Alfvén, 1942), the theoretical understanding of Magnetohydrodynamic (MHD) waves in uniform media of infinite extent rapidly reached substantial sophistication as summarized in the classics by Cowling (1957) and Braginskii (1965). In particular, the role that MHD waves may play in heating the solar atmosphere was pointed out by Cowling as early as in the 1950s (see Cowling, 1962, and references therein). That the structuring in the physical parameters of the solar atmosphere may strongly affect

\section{OPEN ACCESS}

Edited and reviewed by:

Scott William Mclntosh National Center for Atmospheric Research (UCAR), United States

*Correspondence: Tom Van Doorsselaere tom.vandoorsselaere@kuleuven.be

Specialty section:

This article was submitted to Stellar and Solar Physics, a section of the journal Frontiers in Astronomy and Space Sciences

Received: 29 November 2019 Accepted: 24 December 2019 Published: 23 January 2020

Citation: Van Doorsselaere T, Nakariakov VM, Li B and Antolin P (2020) Editorial: Magnetohydrodynamic Waves in the Solar Atmosphere: Heating and Seismology.

Front. Astron. Space Sci. 6:79. doi: 10.3389/fspas.2019.00079 MHD waves was then recognized (e.g., Uchida, 1968; Rosenberg, 1970; Zaitsev and Stepanov, 1975). Even though they paved the way for the now-called "coronal seismology," most modeling studies (e.g., Uchida, 1970; Edwin and Roberts, 1983; Poedts et al., 1990) focused on utilizing the waves for coronal heating (e.g., Ionson, 1978; Hollweg et al., 1982). This modeling was an endeavoring effort, however, because no observational feedback was available, given the lack of instrumental development at the time. Indeed, some scientists even believed that there were no waves in the solar corona. Despite the lack of observational support, many pioneering papers appeared in this subject area.

Substantial indirect evidence suggesting that MHD waves played a key role in explaining the strong emission and broad non-thermal line widths in the upper chromosphere, transition region and corona already existed from observations with Skylab (Feldman et al., 1988), HRTS (Dere and Mason, 1993), and SUMER (Chae et al., 1998). However, there are two key times in transforming the field of coronal wave studies from its early stages to its current level.

The first key discovery was the direct imaging of coronal waves in the SOHO and TRACE era (late 1990s and early 2000s). This came in three important papers that showed the direct evidence of (1) slow waves in footpoints of coronal loops (Berghmans and Clette, 1999) and (2) transverse kink waves in flaring active regions (Aschwanden et al., 1999; Schrijver et al., 1999). The big impact of these papers is in the fact they provided ample feedback on the earlier models for coronal waves. As a result, these discoveries have triggered an avalanche of observational papers on these types of waves, accompanied with detailed analytical and numerical models. Because direct imaging observations of waves suggested insufficient energy for coronal heating (a topic still under debate, see e.g., Terradas et al., 2018), research focus expanded toward their seismological potential (Nakariakov and Ofman, 2001). With coronal seismology, the aim is to use observed wave properties to obtain physical properties of the coronal structure with which the wave is associated, by the comparison with models. 
The second key discovery was the realization that MHD waves are truly omnipresent in the solar atmosphere. This was shown explicitly for the first time with the CoMP instrument (Tomczyk et al., 2007), which only narrowly preceded the Hinode observations (De Pontieu et al., 2007). More recently, a comprehensive analysis of EUV movies of the corona demonstrated the omni-presence of decayless kink oscillations of coronal loops, interpreted as natural standing oscillations continuously sustained by some still debated mechanism (Anfinogentov et al., 2015). These papers have transformed the field, because the observed wave events went from rare observations to space-filling in the corona. Furthermore, the current instrumentation allows to spatially, temporally and spectrally resolve individual aspects of waves. As a result, many researchers are once again considering heating aspects of MHD waves in the solar corona, eschewing somewhat the seismology aspect. Still, it has not been conclusively shown if and how these waves contribute to coronal heating (Arregui, 2015; Hinode Review Team et al., 2019, chapter 6.1).

It must be noted that wave based coronal heating theories are aplenty, and that many of those are theoretically able to produce and maintain a corona (for a review see e.g., Priest, 2014). However, the real challenge lies in identifying key observable predictions from these theories that would allow to discriminate them against observations. This challenge implies, on one hand, conducting advanced numerical simulations of the various wave physical processes-a task that requires enormous amount of computing power due to both, the local and global aspects of wave propagation and dissipation in the heterogeneous solar atmosphere. On the other hand, the challenge requires the synthesis of observable quantities (imaging and spectropolarimetry) from the numerical results, targeting specific instruments and observation conditions-a task known as forward modeling, only possible through the continuous development of atomic databases such as CHIANTI (Dere et al., 1997), and forward modeling codes such as FoMo (Van Doorsselaere et al., 2016).

The historical perspective shows clear trends in the field of MHD waves in the solar atmosphere, in parallel with the development of instrumentation, data analysis techniques, computing power and numerical modeling codes: first there was a strong focus on wave heating, later a strong emphasis on seismology, and now both heating and seismological aspects

\section{REFERENCES}

Alfvén, H. (1942). Existence of electromagnetic-hydrodynamic waves. Nature 150, 405-406. doi: 10.1038/150405d0

Anfinogentov, S. A., Nakariakov, V. M., and Nisticò, G. (2015). Decayless low-amplitude kink oscillations: a common phenomenon in the solar corona? Astron. Astrophys. 583:A136. doi: 10.1051/0004-6361/2015 26195

Arregui, I. (2015). Wave heating of the solar atmosphere. ArXiv e-prints.

Aschwanden, M. J., Fletcher, L., Schrijver, C. J., and Alexander, D. (1999). Coronal loop oscillations observed with the transition region and coronal explorer. Astrophys. J. 520, 880-894. doi: 10.1086/3 07502 and their combination are on the cutting edge of the ongoing research efforts.

The main motivation in organizing this special issue in Frontiers of Astronomy \& Space Sciences is the 20th anniversary of the discovery of waves in the solar corona. Celebrating the first aforementioned key discovery, our aim for this issue was to give an overview of the current efforts in the field, through the display of currently ongoing Research Topics. This issue shows that there is a healthy balance between observational and modeling papers in the field of MHD waves in the solar atmosphere, following the larger trend in solar physics. Moreover, the two main directions in the field are reflected very well in this content collection: using the observed MHD waves for coronal seismology and coronal heating. This shows perhaps that a new balance has been found within the community between these two important research directions.

In the current special issue, we see also an accent on the basic understanding of wave phenomena in MHD and beyond. This shows two aspects: (1) Wave behavior in MHD is not completely understood yet at a theoretical level, and (2) a significant effort is now being done to understand what is happening beyond MHD (e.g., two fluids). This opens up potential for even more applications in the solar atmosphere, and indicates that there is a lot of room to expand, perhaps by focusing on the smaller scales that are not accessible by MHD waves.

\section{AUTHOR CONTRIBUTIONS}

TV wrote the initial draft. PA and BL added several paragraphs. VN gave comments.

\section{FUNDING}

TV was supported by the European Research Council (ERC) under the European Union's Horizon 2020 research and innovation programme (grant agreement No. 724326) and the C1 grant TRACEspace of Internal Funds KU Leuven. PA acknowledges funding from his STFC Ernest Rutherford Fellowship (No. ST/R004285/1). VN acknowledges funding from STFC consolidated grant ST/P000320/1, and the Russian Foundation for Basic Research Grant No. 18-29-21016. BL was supported by the National Natural Science Foundation of China (41674172 and 11761141002).
Berghmans, D., and Clette, F. (1999). Active region euv transient brightenings - first results by eit of soho jop80. Solar Phys. 186, 207-229. doi: 10.1023/A:1005189508371

Braginskii, S. I. (1965). Transport processes in a plasma. Rev. Plasma Phys. 1:205. doi: 10.1007/978-3-319-62006-0_3

Chae, J., Schühle, U., and Lemaire, P. (1998). SUMER measurements of nonthermal motions: constraints on coronal heating mechanisms. Astrophys. J. 505, 957-973. doi: 10.1086/306179

Cowling, T. G. (1957). Magnetohydrodynamics. New York, NY: Interscience Publishers.

Cowling, T. G. (1962). Magnetohydrodynamics. Rep. Prog. Phys. 25, 244-286.

De Pontieu, B., McIntosh, S. W., Carlsson, M., Hansteen, V. H., Tarbell, T. D., Schrijver, C. J., et al. (2007). Chromospheric Alfvénic waves strong enough 
to power the solar wind. Science 318, 1574-1577. doi: 10.1126/science.11 51747

Dere, K. P., Landi, E., Mason, H. E., Monsignori Fossi, B. C., and Young, P. R. (1997). CHIANTI - an atomic database for emission lines. Astron. Astrophys. Suppl. Ser. 125, 149-173. doi: 10.1051/aas:1997368

Dere, K. P., and Mason, H. E. (1993). Nonthermal velocities in the solar transition zone observed with the high-resolution telescope and spectrograph. Solar Phys. 144, 217-241. doi: 10.1007/BF00627590

Edwin, P. M., and Roberts, B. (1983). Wave propagation in a magnetic cylinder. Solar Phys. 88, 179-191.

Feldman, U., Doschek, G. A., and Seely, J. F. (1988). Solar spectroscopy in the farultraviolet-X-ray wavelength regions - Status and prospects. J. Opt. Soc. Am. B Opt. Phys. 5, 2237-2251. doi: 10.1364/JOSAB.5.002237

Hinode Review Team, Al-Janabi, K., Antolin, P., Baker, D., Bellot Rubio, L. R., Bradley, L., et al. (2019). Achievements of Hinode in the first eleven years. Publ. Astron. Soc. Jpn. 71:R1. doi: 10.1093/pasj/psz084

Hollweg, J. V., Jackson, S., and Galloway, D. (1982). Alfven waves in the solar atmospheres - Part three - Nonlinear waves on open flux tubes. Solar Phys. 75, 35-61. doi: 10.1007/BF00153458

Ionson, J. A. (1978). Resonant absorption of Alfvénic surface waves and the heating of solar coronal loops. Astrophys. J. 226, 650-673. doi: 10.1086/156648

Nakariakov, V. M., and Ofman, L. (2001). Determination of the coronal magnetic field by coronal loop oscillations. Astron. Astrophys. 372, L53-L56. doi: 10.1051/0004-6361:20010607

Poedts, S., Goossens, M., and Kerner, W. (1990). Temporal evolution of resonant absorption in solar coronal loops. Comput. Phys. Commun. 59, 95-103. doi: 10.1016/0010-4655(90)90159-X

Priest, E. (2014). Magnetohydrodynamics of the Sun Cambridge, UK: Cambridge University Press.

Rosenberg, H. (1970). Evidence for MHD pulsations in the solar corona. Astron. Astrophys. 9:159.
Schrijver, C. J., Title, A. M., Berger, T. E., Fletcher, L., Hurlburt, N. E., Nightingale, R. W., et al. (1999). A new view of the solar outer atmosphere by the Transition Region and Coronal Explorer. Solar Phys. 187, 261-302.

Terradas, J., Magyar, N., and Van Doorsselaere, T. (2018). Effect of magnetic twist on nonlinear transverse kink oscillations of line-tied magnetic flux tubes. Astrophys. J. 853:35. doi: 10.3847/1538-4357/aa9d0f

Tomczyk, S., McIntosh, S. W., Keil, S. L., Judge, P. G., Schad, T., Seeley, D. H., et al. (2007). Alfven waves in the solar corona. Science 317, 1192-1196. doi: 10.1126/science.1143304

Uchida, Y. (1968). Propagation of hydromagnetic disturbances in the solar corona and Moreton's wave phenomenon. Solar Phys. 4, 30-44. doi: 10.1007/BF00146996

Uchida, Y. (1970). Diagnosis of coronal magnetic structure by flare-associated hydromagnetic disturbances. Publ. Astron. Soc. Jpn. 22:341.

Van Doorsselaere, T., Antolin, P., Yuan, D., Reznikova, V., and Magyar, N. (2016). Forward modelling of optically thin coronal plasma with the fomo tool. Front. Astron. Space Sci. 3:4. doi: 10.3389/fspas.2016.00004

Zaitsev, V. V., and Stepanov, A. V. (1975). On the rigin of pulsations of type IV solar radio emission. Issled. Geomagn. Aeron. Fiz. Solntsa Vyp, 3-10.

Conflict of Interest: The authors declare that the research was conducted in the absence of any commercial or financial relationships that could be construed as a potential conflict of interest.

Copyright (C) 2020 Van Doorsselaere, Nakariakov, Li and Antolin. This is an openaccess article distributed under the terms of the Creative Commons Attribution License (CC BY). The use, distribution or reproduction in other forums is permitted, provided the original author(s) and the copyright owner(s) are credited and that the original publication in this journal is cited, in accordance with accepted academic practice. No use, distribution or reproduction is permitted which does not comply with these terms. 